La houlle noire a fait lindustrie moderne; la houille blanche la transformera.

\section{CONSIDÉRATIONS PRATIQUES} SUR L'EXPLOITATION DES BREVETS D'INVENTION

\section{$-($ SUTTE $)-$}

\section{La contrefaçon. - Sa constatation. - Sa poursuite Sa répression.}

Fanli de son brevet et en possession du titre qui lui garantil un droit privalif, l'inventeur n'est pas toujours au boul de ses peines et n'a pas toujours surmonté toutes les difficultés qui peuvent se présenter sur sa route.

La loi lui fail une obligation impérieuse d'exploiter son brevet, sanctionnée par la déchéance à défaut d'exploitation dans les trois ans à dater du jour de la demande.

Son intérêt lui commande d'exploiler.son brevel. Ge n'est pas toujours facile : le breveté peut se heurter à des difficullés, notamment à des difficultés d'ordre financier.

Le breveté peut être paralysé dans son exploitalion par des actes de contrefaçon.

Sous peine d'être privé du fruit de son invention, ou de ne le recucillir que d'une façon incomplète, le breveté doil poursuivre la contrefaçon et la poursuivre d'autant plus rapidement et d'autant plus énergiquement que son monopole n'a qu'une durée limitée $(1)$.

Supposons qu'un inventeur breveté estime qu'un tiers usurpe son invention et pratique la contrefacon à son détriment. Comment peut-il saisir la contrefaçon ? Comment peut-il en rapporter la preuve? Quelle procédure doit-il suivre? Quels sont les incidents qui peuvent se produire a'u cours de cette procédure? Qucls en sont les risques et les dangers?

Lo breveté qui est victime de contrefaçon et qui veut en poursuivre la répression doit en rapporter la preuve : il peut faire la preuve par tous les moyens.

Si le brevet porte sur un produit, sur un appareil, le breveté peut en faire l'acquisition chez le présumé contrefacteur en s'entourant des garanties nécessaires pour quo l'origine et l'identité du produit ou de l'appareil ne soient pas contestables.

Le brevel peut garantir un objet non susceptible d'appréhension : un procédé de fabrication, par exemple.

lic breveté apprend qu'un industricl exploite le procédé qu'il a trouvé, à l'abri des indiscrétions, derrière les murs d'une usine, donl la porte est soigneusement consignée aux roncurrents et aux curieux.

Comment fera-t-jl pour se procurer la preuve de la ontrefaçon?

Le brevelé prut en faire constater lexistence dans les conditions spéciales prévues par l'articlo 17 de la loj de $x 84 / 4$ qui instilue deux modes spéciaux de constatation judiciare : la description et la saisie.

la description est un constat : elle laisse l'objet décrit à

(1) La Commission nommée pour examiner le projet de loi portant modiflation de la loi de 1844 , propose de porter à.vingt ans la dur'e des brevets par modificatio. de l'article 4 de la loi 1844. la disposition du présumé contrefacteur, elle en précise et en analyse les caracteres et les étéments pour permettro aux juges d'opérer ensuile la comparaison entre l'objel décrit et le brevet.

La saisie est beaucoup plus grave, elle constilue uno main-mise sur l'objet présumé contrefail, elle le confisque provisoirement, soit qu'il soit mis sous scellés et daus l'impossibilité de fonctionner, soit qu'il soil déposé au greftie du tribunal ou en fourrière.

L'article 47 de la loi de 1844 qui prévoit la procédure à suivre pour la constatation de la contrefaçon el sa répression dispose de la façon suivante :

" Les propriélaires de brevet pourront, en vertu d'unc ordonnance du Président du Tribunal de première instance, faire procéder par tous huissiers à la désignalion el description détaillées, avec ou sans saisie, des objets prétendus controfaits.

"L'ordonnance sera rendue sur simple requete el sur la représentation du brevet : elle contiendra s'il y a lieu la nomination d'un expert pour aider l'huissier dans sa description.

"Lorsqu'il y aura lieu à saisie, la dite ordonmance pouma imposer au requérant un cautionnement qu'il sera tenu de consigner avant d'y faire procéder.

" Le cautionnement sera toujours imposé à l'ćtranger breveté qui requerra la saisie.

"Il sera laissé copie au détenteur des objets dícrits ou saisis, tant de l'ordonnance que de l'acte constatant le dépôt du cautionnement, le cas échéant : le tout, à peine de nullité et de dommages-intérêts contre l'huissier $"$.

Supposons que le breveté sollicile du Président du Tribunal de première instance l'ordonnance nécessairc.

Suffit-il de présenter une demande accompagnóe du brevet et de l'indication que le brevet cst l'objet de contrefaçon pour obtenir l'autorisalion de décrire, de saisir, etc. ?

La jurisprudence accorde au Président du Tribunal un pouvoir souverain, en ce sens qu'il ne peut pas refuser d'autoriser une description, mais qu'il est juge du point de savoir s'il doil autoriser une saisie, el si cclle saisic, dans les cas où la chose est possible, peut être faite sans cautionnement préalable.

L’ordonnance rendue par le Président, qui autorje l'entréc dans une usine peut avoir des effels langereux el irréparables dans le cas où le breveté, arguant d'une contrefaçon, se servirait de la procúlure de la loi de 18 /4 pour aller surprendre un secrel de fabrique, se rendre compte d'une installation d'usine, d'une disposition d'éléments.

Le Président ne rendra son ordonnance qu'après avoir pris en considération la personne du plaignant of sa siluation, la personne el la situation du présumé contrefacteur, limportance du brevel, la notoriété de la contrefaçon.

Il mettra à l'exécution de son ordonnance les réserves que prévoit la loi de 1844 , mais il ne peut pas refuser de rendre une ordonnance qui peut êre préjudiciable au tices qui en est l'objet of qui peut engager, le cas échéant, la responsabilité de celui qui l'a sollicitéc of qui en fait usage.

Supposons que le breveté suive la procédure indiquós par l'article 47 de la loi de $84 / \mathrm{f}$ ef qu'il obtienne wre ordon- 
nance générale du type de l'ordonnance suivante, rendue par le Président du Tribunal Civil de Lyon, à la requête de la Société la liuchsine, le 9 août 1864 .

\section{« Nous, Président du Tribunal Civil de Lyon, etc. :}

Vu la requête qui précède et les brevets sus-énoncés ; - Vu l'article 47 de la loi du 5 juillet 1844 ; - Nutorisons les exposants à faire procéder individuellement ou collectivement dans toute l'étendue de l'arrondissement de Lyon, par le premier huissier, de ce requis, avec l'assistance, s'il y a lieu, de tout commissaire de police et mème de la force publique, soit au domicile, soit sur la voie publique, chez ou au préjudice de tout fabricant, marchand, apprêteur, teinturier, détenteur, colporteur, entrepositaire, dépositaire public ou privé, ainsi que dans les gares de chemin de fer et leurs dépendances, bureaux de messageries et de roulage, quais d'arrivage par eau, bureaux de douane, et enfin partout où besoin sera, même en cas d'expédilion en transit, à là saisie : $x^{0}$ de diverses matiòres colorantes, rouge, rose, saumon, bleu, violet et marron, dont s'agit, ainsi que celles qui en sont extraites, blanc, vert, grenat et autres; $2^{\circ}$ des étoffes matières et objets quelconques, teints ou imprimés au moyen des produits contrefaits ou argués comme eux de contrefaçon, ainsi que des factures, lettres de voiture, livres, correspondances et papiers y relatifs; $3^{\circ}$ des instruments, ustensiles, appareils, matières premières servant à la fabrication des produits contrefaits, ainsi que ceux de ces produits en cours de fabrication; - Ordonnons que, suivant les circonstances, les objets saisis seront enlevés et déposés au greffe, ou transportés ou laissés dans les divers entrepòts publics, bureaux de douane, gares de chemin de fer ou à la garde des gardiens forcés choisis parmi les saisissants ; ordonnons qu'il sera procédé dans les lieux sus-indiqués et partout où besoin sera à toutes recherches et perquisitions, à l'effet de découvrir la contrefaçon et les produits argués de contrefaçon, à toutes recherches et perquisitions sur les livres, écritures, papiers, dans le but de constater l'étendue et l'importance de la contrefaçon et suivant les circonstances, à parafer ne varietur, sans déplacement, ou à enlever et déposer au greffe lesdits livres et papiers; - Aux effets ci-dessus, aulorisons les exposants ì se faire représenter aux dites opérations par le directeur de la Fuchsine ou son représentant et autorisons l'huissier à se fáire assister, s'il y a lieu, par un expert chimiste. Commettons à cet effet, comme expert, M. Ferrand, pharmacien, dispensé du serment ;

Ordonnons l'exécution de notre ordonnance, même les jours fériés et hors des heures ordinaires des exploits ; - Disons qu'en cas de référé, il sera préalablement procédé à la saisie des matières colorantes arguées de contrefaçon ot des objets quelconques, teints ou colorés avec elles, ainsi qu'à la saisie des papiers ou examen et parafe des livres et écritures et autres perquisitions de nature à constater la quantité dè objels saisissables et l'importance de la contrefaçon.

Muni de pareille autorisation, le breveté se présente en personne à la porte de l'usine où il estime que se pratique la contrefaçon, accompagné d'un huissicr, d'un expert et du commissaire de police. La porte s'ouvre : le breveté pénètre accompagné de ses auxiliaires ct se livre aux investigations autorisées.

Le breveté fait décrire le procédé exploité, les âppareils employés, leur marche et le résultat qu'ils fournissent, fait saisir les appareils ainsi que des échanlillons des produits fabriqués, qui sont mis sous scellés et cachetés de façon à assurer leur identité. II examine la comptabilité qui est paraphée ne varielur, il saisit la correspondance el les factures.

L'huissier dresse un procès-verbal des opérations, procèsverbal qui est remis au tiers objet de la visite. Le breveté a trouvé la preuve de la contrefaçon, il va en poursuivre la répression et la réparation du préjudice qu'il en a subi, en assignant, à peine de nullité, dans la huitaine, devant le Tribunal civil ou devant le Tribunal correctionnel.
L'opération si complète que vient d'exécuter le breveté, par hypothèse, ne s'exécutera pas, en général, sans protestations de la part du présumé contrefacteur.

Il peut tout d'abord refuser l'entrée de son usine à l'huissier et aux personnes qui l'accompagnent, désignées par l'ordonnance. L'huissier doit alors requérir le Juge de Paix ou le Commissaire de police : assisté de ces magistrats, il peut procéder à des constatations régulières.

A défaut d'assistance du Juge de paix ou du Commissaire de police, la pénétration dans l'usine constituerait une violation de domicile.

Ainsi décidé par un arrêt de la Cour de Paris du $\mathbf{x} 2$ décembre 1856 , rendu dans les circonstances suivantes.

Le 14 février I 855 , M. L..., dûment autorisé par une ordonnance du Présidenl du Tribunal civil de Brest, se présente dans l'usine de M. T..., fabricant de produits chimiques au Conquet, accompagné du Juge de paix et d'un huissier. M. T... proteste, déclare qu'il ne contrefait aucun produit, que la visite révèlera à M. L... des secrets de fabrique. M. L... persiste, pénètre dans l'usine et, après examen des machines et des produits, conslate que rien ne peut faire soupçonner M. T... de contrefaçon.

M. T... assigne M. L... en 25.000 franos de dommagesintérêts devant le Tribunal de la Seine qui, par jugement du 23 janvier 1856 confirmé par arrêt du 12 décembre 1856 , statue dans les termes suivants sur la validité des opérations.

"Attendu que, d'un procès-verbal du 14 février I 855 , il résulte que $L . .$. en vertu d'une ordonnance du Président du Tribunal de Brest, assisté du Juge de paix du canton, s'cst présenté à l'usine T... au Conquet, à l'effet de constater si ce dernier se servait, pour la fabrication de ses produits chimiques, de machines et d'appareils conformes à ceux faisant l'objet des brevets dont il est propriétaire.

"Attendu que, malgré l'opposition de T..., et sa protestation qu'il n'avait rien contrefait, L... a pénétré dans la cour intérieure de la fabrique avec l'huissier et les témoins et, après s'être assuré qu'il ne voyait aucune trace de produits ammoniacaux, il a renoncé à continuer ses perquisitions et s'est retiré sans pratiquer de saisie et sans faire de description des procédés et appareils servant à la fabrication des produits de T....

"Attendu que ces faits ne peuvent constituer une violalion de domicile. "

Le présumé contrefacteur peut rofuser l'entrée de son usine au brevelé, à moins que l'ordonnance rendue conformément à l'article 47 de la loi de 1844 n'autorise Je breveté à assister aux opérations.

Le breveté a un intérêt considérable et facile à comprendre, à suivre en personne les opérations de description et de saisie. Mais sa présence chez un concurrent peut être nujsible à ce dernicr, car à la faveur du droit que lui confère l'ordonnance rendue, il peut surprendre des secrets de fabrique, examiner des machines el des appareils dont il ignorait l'emploi, l'existence et le fonclionnement.

Supposons qu'après avoir triomphé de ces premières difficullés, le breveté accompagné de ses auxiliaires légaux, pénètre dans l'usine.

L'ère des difficultés n'est pas close.

Le présumé contrefacteur peut et doit exiger Jpcture ét copic de l'ordonnance du président autorisant la description ou la saisie et indiquant les conditions auxquelles ce magistrat subordonne l'exécution des opérations. 
Le présumé contrefactour peut alors et suivant les cas, soutenir' une série de moyens contre l'exécution de l'ordomnance.

Il peut soutenir qu'il n'y a pas lieu à saisie, qui paralyse d'une façon inutile son exploitation et qu'une description est suffisante pour garantir les intérêts que veut défendre le breveté.

Il peut soutenir, en cas de contrefaçon de produit, qu'il esl inutile de saisir une série de produits et quo la saisie d'un seul produit est suffisante pour permettre de rapporter, le cas échéant, la preuve de la contrefaçon.

Il peut soutenir que le breveté doit fournir un cautionnement préalablement à la saisie.

Il peut s'opposer à la saisie de la correspondance et des facturcs, soutenant qu'elle est illégale et qu'elle ne peut pas atre aulorisée par le Président du Tribunal dont l'ordonnance est, de ce chef, entachée d'excès de pouvoir.

Ces protestations sont-elles de nature à empêcher ou à suspendre les opérations?

Les ordonnances autorisant perquisition ou saisie, sont rendues en général à charge de référ er en cas de difficultés. l. breveté saisira le Président statuant en état de référé pour fance vider l'incident.

Les opérations seront suspendies en principe jusqu'à la solulion du référé, sauf dans le cas où l'ordonnance conliendra la formule suivante qu'il est prudent, pour celui qú poursuit la contrefaçon, de faire insérer : "Disons qu'en cas de difficultés, il nous en seria référé, mais après constatation préalablement faite ". Dans ce cas, les protestalions n'empêcheront pas l'exécution de l'ordonnance et, le ras ćchéant, la constatation de la contrefaçon.

(à suivre)

A. Bugand.

Avocat à la Cour d'Appel de Lyon.

\section{ÉCONOMIE MONTAGNEUSE}

\section{UN PROBLÈME SOCIAL}

DANS LES MONTAGNES MÉRIDIONALES FRANGAISES

Sur l'ensemble des régions françaises en proie à la dépopulation contemporainc, le groupe de nos $3 \mathrm{r}$ départements monlagneux du Midi est celui qui paie depuis longtemps le plus lourd tribut au fléau. I a situation généralement très accidentée et l'onsemble des autres conditions géographiques de ces pays méridionaux y prédisposent le sol aux dégradalions torrentielles ; celte siluation y impose le plus souvent la cullure pastorale dont les abus provoquent toujours celte dégradation, surtout, comme c'est le cas, quand le mouton domine dans le capital pécoral ; enfin les conditions naturellement difficiles de la vic montagneuse y poussent les habitants aux migrations, aux dépaysements. Il est donc légitime de chercher dans des contingences géographiques, ćconomiques el sociales, les éléments d'un Probleme social que ne se sont pas explicitement posé jusqu'ici les restaurateurs du sol montagneux; ef qui doit viser plus que jamais aujourd'hui la stabilisation et l'évolution progressive de nos montagnards. Discuter largement et attentivement ces questions est d'autant plus opportun qu'une législation

(1) Analrse d'un mémoire nrésenté par l'auteur à la a Section des sciences pronnminnes et sociales du 510 Congrés fles socibtés savantes o à Crenoble le 13. mai 1919, pn rénonse à la deuxième queslion dn nrogramme: Rechercher dans une region les causes économiques de la desertion des campagneset les movens suscotibles d'en arretcr le mourement. monlagneuse nouvelle dont les effels sociaux peuvent être désastreux, est actucllement pendante au Parlement.

Pour la masse de nos monlagnards méridionaux, le déricinement équivaut aujourd'hui à l'expalrialion el le plus souvent à la dénationalisalion (1) : leur retour au pays est des plus problématiques. Des travailleurs étrangers se substituent aisément à eux, šinstallent même souvent sur leurs " coins de terre " délaissés. Une des raisons malérielles et capilales de celle évasion se trouve dans la dégénérescence, en qualité et en quantité, des anciens communaux sur lesquels s'alimentait le bélail, matic̀re de vie de ces populations pastorales. Les abus de l'élevage extensif imhérents en principe à la jouissance collective et qui ne peut y che aulre, ont évincé bêtes et gers de pâturages stérilisés et lorrentialisés pour longtemps. Les énergies du pays se trouvent gravement alteintes par ce double jeu d'évasion métropolilaine ol d'invasion étrangère.

Ia colonisation officielle en Algéric a assumé une large part de responsabilité dans cette crise. C'est elle qui organisa l'exode il y a 30 ans quand, en suile de la législation monlagneuse du 4 avril $188 \%$, débuta la nationalisalion des sols à restaurer ${ }^{(2)}$. L'Etat était alors bien décidé, quoique déjà pleinement conscient des dévastations matérielles, à limiter la restauration projetée aux seuls territoircs absolument dégradés, sans prendre, à l'égard des autres, aucune mes'ure préventive. Il a jusqu'à présent nationalisé, par cxpropriation ou acquisition amiable, plus de 200.000 hectares, en grande partie communaux. Les doléances des nombreuses familles qui les pcuplaient, et dont certaines comptaient plus de ro personnes, furent apaisées avec des lots de colonisation de terrains expropriés ou simplement confisqués aux Arabes. La colonisation officielle est aujourd'hui condamnée par tout le monde, même dans la petite patrie. Mais, en France, la nationalisation du sol fonclionne toujours, à raison de cinq à six mille hectares par an ; on la stimule, il faut bien dépenser les crédits ! C'est, au bas mot, une centaine de familles rurales de la grande palrie, que l'Etat voue ainsi et très chèrement, au prix de plus de 500 ooo fr., i la dénationalisation.

C'est à un pur mirage hydrologique que le pays est redevable de cet étrange et coutleux procédé de restauration monlagneuse... au moyen du vide I Les lechniciens d'il y a cinquante ans, hantés par l'idée que le roboisoment des montagnes qu'on avait mis des siecles à dénuder sans que nul n'en prît cure, garantivait j̀ jamais nos plaines d'inondation's désastreuses qui sont et resteront probablement dans le tempéramment de corlains grands lleuves, pour ne pas dire de tous, conduisirent le législateur, par une mésadaptation absolue de la loi de 18 / r sur les "grands travaux " d'utilité publique, et en vue d'une problématique sécurilé à assurer aux gens de l'aval, à exproprier ceux de l'amont trop obstinés à refuser les futurs bienfaits d'un reboisement auxquels eux et leurs troupeaux avaient immódiatement foul ¿ perdre. Une expérience de tronte ans réaliséc au prix do cent millions de francs, a mis en complète faillite celte restauration où les grands travinx architectoniques prirent une part démesurée ; dle a clairement montré les dangers sociaux provoqués par cefte spéculation hydrologique qui, dans certaines régions très forrentielles, n'a guère cu du aboisemant que le nom.

(1) Levasion contemporaine des montaynarls franeals. (Annales de la seience agronnmique française et ctrangere. Janvier $1911,0,1-51)$.

(A) Restauration et nalionalisation du sol en have montagne, $(A$, . A. S. $1911, p \cdot 1060-1066)$. 